15,14

\title{
Термомеханический режим формирования вихревых течений в гибридно ориентированном нематическом канале
}

\author{
(C) A.B. Захаров \\ Институт проблем машиноведения РАН, \\ Санкт-Петербург, Россия \\ E-mail: alexandre.zakharov@yahoo.ca
}

Поступила в Редакцию 15 января 2019 г. В окончательной редакции 15 января 2019 г.

Принята к публикации 15 января 2019 г.

\begin{abstract}
Численными методами, в рамках нелинейного обобщения классической теории Эриксена-Лесли, допускающей учет термомеханических вкладов как в выражение для сдвигового напряжения, так и в уравнение баланса энтропии, описаны несколько сценариев формирования вихревых течений (BT) в микроразмерных гибридно-ориентированных жидкокристаллических (ГОЖК)-каналах с ориентационными дефектами. Анализ численных результатов показал, что ГОЖК-канале два либо один вихрь, хотя на начальном этапе формирования ВТ зарождаются два вихря, направленные навстречу друг другу.
\end{abstract}

Работа выполнена при финансовой поддержке Минобрнауки (гранты 3.11888.2018/11.12 и 3.9585.2017/8.9).

DOI: 10.21883/FTT.2019.06.47699.358

\section{1. Введение}

В последнее время все чаще методы микро- и нанофлуидистики находят применение не только при исследовании процессов транспортировки и сортировки нанолитровых объемов молекулярных жидкостей [1-4], но и в разнообразных семействах сенсоров и датчиков на основе термо- и лиотропных жидкокристаллических (ЖК) материалов [3,5]. Еще одной не менее интригующей областью применения микролитровых объемов ЖК-материалов являются биометрические оптические системы, где основным элементом являются ЖК-линзы с регулируемым фокусным расстоянием (tunable liquid crystal microlenses) [6,7]. Один из способов, позволяющий манипулировать такими микроразмерными молекулярными системами, основывается на использовании электрического поля [8]. Другой способ, позволяющий транспортировать микролитровые объемы молекулярных жидкостей, основан на формировании разности градиентов поверхностного натяжения (ГПН) на границах раздела жидкость/газ и жидкость/твердое тело [9]. Эта разность ГПН может быть создана посредством локального разогрева ограниченного объема жидкости, например, с помощью сфокусированного лазерного излучения. Эти вышеописанные методы транспортировки микролитровых объемов одинаково применимы как для молекулярных жидкостей, так и для манипулирования микролитровыми объемами ЖК-материалов. Отличительной особенностью ЖК-систем от изотропных молекулярных жидкостей является то, что при определенных термодинамических условиях в ЖК-системах формируется ориентационное упорядочение молекул, которое описывается полем директора $\hat{\mathbf{n}}$ [10]. Было показано, что взаимодействие градиентов поля директора $\nabla \hat{\mathbf{n}}$ и температуры $\nabla T$ ведет к формированию устойчивого гид- родинамического потока $\mathbf{v}$ ЖК-материала [11]. Величина этого потока пропорциональна сдвиговой составляющей термомеханического вклада $\sigma_{z x}^{t m}$ в тензор напряжения (ТН) $v \sim \frac{d}{\eta} \sigma_{z x}^{t m}$, где $\sigma_{z x}^{t m} \sim \xi \frac{\Delta T}{d^{2}}, \eta-$ сдвиговая вязкость ЖК-материала, $\Delta T=T_{2}-T_{1}>0$, а область температур $\left[T_{2}, T_{1}\right]$ находится в пределах стабильности нематической фазы толщиной $d$, в то время как $\xi-$ термомеханическая постоянная [12]. Если локальный градиент температуры в объеме ЖК-фазы легко формируется с помощью лазерного излучения, то градиент поля директора удается сформировать посредством гибридной ориентации ЖК (ГОЖК)-фазы, в которой ориентация поля директора на одной из поверхностей планарная, а на другой - гомеотропная. Все это указывает на то, что существует возможность немеханической транспортировки микролитровых объемов ЖК-материала под действием сфокусированного лазерного излучения. Но нужно принять во внимание тот факт, что в микроразмерных объемах ЖК-материала всегда присутствует какое-то количество ориентационных дефектов (ОД), которые влияют на процесс транспортировки ЖК-материалов. Степень влияния ОД, а также величины и направления теплового потока q, инициируемого лазерным излучением на характер возбуждаемого гидродинамического течения в микроразмерном ЖК-канале, будет исследована в рамках нелинейного обобщения классической теории Эриксена-Лесли $[13,14]$, с учетом баланса энтропии [15].

\section{2. Основные уравнения}

Рассмотрим длинный прямоугольный ЖК-канал с размерами $2 D$ и $2 d(D \gg d)$, ограниченный твердыми горизонтальными и вертикальными поверхностями. Допустим, что директор планарно ориентирован на верх- 
ней и двух боковых поверхностях, и гомеотропно на нижней поверхности. Рассмотрим систему координат, отсчитываемую от середины ЖК-канала так, что ось $X$ и орт $\hat{\mathbf{i}}$ совпадают с направлением директора на верхней ограничивающей поверхности $\left(\hat{\mathbf{i}} \| \hat{\mathbf{n}}_{z=d}\right)$, в то время как ось $Z$ и орт $\hat{\mathbf{k}}$ направлены ортогонально $\left(\hat{\mathbf{k}} \perp \hat{\mathbf{n}}_{z=d}\right)$, а орт $\hat{\mathbf{j}}=\hat{\mathbf{k}} \times \hat{\mathbf{i}}$. Будем предполагать, что переориентация поля директора $\hat{\mathbf{n}}=\left(n_{x}, 0, n_{z}\right)=\sin \theta \hat{\mathbf{i}}+\cos \theta \hat{\mathbf{k}}$ под действием градиента температуры $\nabla T$, формирующегося за счет сфокусированного лазерного излучения, осуществляется в плоскости $X Z$. Здесь $\theta \equiv \theta(x, z, t)$ - полярный угол, образованный директором $\hat{\mathbf{n}}$ и ортом $\hat{\mathbf{k}}$. Далее мы будем использовать безразмерные пространственные координаты $\bar{x}=\frac{x}{d}$ и $\bar{z}=\frac{z}{d}$, причем в дальнейшем верхняя черта будет опущена.

В настоящей работе предлагается новый подход описывающий механизм формирования вихревого течения в микроразмерном ГОЖК-канале под действием потока тепла q, направленного под углом $\alpha$ к нижней ограничивающей поверхности канала, содержащей ориентационный дефект. Этот ОД характеризуется непрерывным изменением поля директора от гомеотропной ориентации к планарной, и обратно к гомеотропной. Таким образом, вдоль нижней ограничивающей поверхности ГОЖК-канала формируется градиент $\nabla \theta$, то есть

$$
\begin{gathered}
\theta_{-10 \leq x \leq-L, z=-1}=\theta_{L \leq x \leq 10, z=-1}=0, \\
\theta_{-L<x<L, z=-1}=\mathcal{A},
\end{gathered}
$$

где $\mathcal{A}=\tan ^{-1}\left(\frac{L^{2}-x^{2}}{4 x^{4}}\right), L=\frac{l}{d}$, и $2 l-$ линейный размер ОД на нижней ограничивающей поверхности, в то время как на остальных поверхностях директор ориентирован планарно, то есть

$$
\theta_{x= \pm 10,-1<z<1}=0, \quad \theta_{-10 \leq x \leq 10, z=1}=\frac{\pi}{2} .
$$

В дальнейших расчетах будем считать, что отношение $D / d$ равно 10. Для того чтобы понять, какую роль играет тепловой поток $\mathbf{q}=q_{z} \hat{\mathbf{k}}=\mathscr{Q} \sin \alpha \hat{\mathbf{k}}$ в формировании вихревого течения в микроразмерном ГОЖК-канале, предположим, что лазерное излучение сфокусировано на нижней ограничивающей поверхности содержащей ориентационный дефект. Здесь $\alpha-$ угол образованный вектором $\hat{\mathbf{q}}$ и ортом $\hat{\mathbf{i}}$, а функция

$$
\mathscr{Q}(x, z)=\mathbb{Q}_{0} \exp \left(-2 \frac{\left(x-x_{0}\right)^{2}+\left(z-z_{0}\right)^{2}}{\Delta}\right) \mathscr{H}\left(\tau_{\text {in }}-\tau\right)
$$

описывает распределение инжектируемой энергии в микроразмерном ГОЖК-канале, в то время как $\mathbb{Q}_{0}$ - безразмерная мощность теплового потока, $\Delta=\frac{\omega_{0}}{d}=2 L-$ размер Гауссового пятна лазерного излучения, $x_{0}$ и $z_{0}-$ координаты его центра соответственно, $\mathscr{H}\left(\tau_{\text {in }}-\tau\right)-$ функция Хэвисайда, $\tau=\frac{t}{t_{R}}-$ безразмерное время в течении которого протекает процесс формирования вихревого течения, $\tau_{\text {in }}-$ характерное безразмерное время накачки лазерного излучения в ЖК-фазу.
Граничное условие для безразмерной температуры $\chi \equiv \chi(x, z, \tau)=T(x, z, \tau) / \mathrm{NI}$ может быть записано в виде

$$
\left(\chi_{, z}(x, z, \tau)\right)_{z=-1}=\left[\frac{q_{z}-(\lambda-1) n_{x} n_{z} \chi, x}{\lambda n_{z}^{2}+n_{x}^{2}}\right]_{z=-1},
$$

в то время как температура на остальных ограничивающих поверхностях постоянна и равна

$$
\chi_{-10 \leq x \leq 10, z=1}=\chi_{x \pm 10,-1<z<1}=\chi_{c} .
$$

Здесь $\lambda=\lambda_{\|} / \lambda_{\perp}, \lambda_{\|}$и $\lambda_{\perp}-$ коэффициенты теплопроводности ЖК-фазы в направлении параллельном и перпендикулярном направлению директора $\hat{\mathbf{n}}, \chi_{, x}=\frac{\partial \chi}{\partial x}$, и $T_{\mathrm{NI}}-$ температура фазового перехода нематик-изотропная фаза соответственно. В нашем случае поле скорости $\mathbf{v}=u \hat{\mathbf{i}}+w \hat{\mathbf{k}}$ удовлетворяет граничным условиям

$$
\begin{aligned}
& u_{-10 \leq x \leq 10, z=-1}=u_{x= \pm 10,-1<z<1}=0, \\
& w_{-10 \leq x \leq 10, z=-1}=w_{x \pm 10,-1<z<1}=0,
\end{aligned}
$$

где $u \equiv v_{x}(x, z, \tau)$ и $\left.w \equiv v_{z} m x, z, \tau\right)$ - являются компонентами вектора скорости v. Принимая во внимание тот факт, что толщина ЖК-канала варьируется в пределах нескольких микрометров, будем считать, что плотность ЖК-фазы постоянна по сечению канала ( $\rho=$ const). Таким образом, мы имеем дело с несжимаемой ЖК-фазой, и условие несжимаемости $\nabla \cdot \mathbf{v}=0$, которое принимает вид

$$
u_{, x}+w_{, z}=0,
$$

выполняется за счет введения функции тока $\psi$, где $u=\psi_{, z}$, а $w=-\psi_{, x}$ соответственно.

В нашем случае безразмерные уравнения баланса угловых и линейных моментов, действующих на единицу объема ЖК-фазы, а также баланса энтропии имеют вид (см. Приложение и ссылку [16])

$$
\begin{aligned}
& n_{z} n_{x, \tau}-n_{x} n_{z, \tau}=\delta_{1}\left[n_{z} \mathscr{M}_{0, x}-n_{x} \mathscr{M}_{0, z}\right. \\
& \left.+K_{31}\left(n_{z} f_{, z}+n_{x} f_{, x}\right)\right]-\frac{1}{2} \psi_{, x x}\left[1+\gamma_{21}\left(n_{x}^{2}-n_{z}^{2}\right)\right] \\
& -\frac{1}{2} \psi_{, z z}\left[1-\gamma_{21}\left(n_{x}^{2}-n_{z}^{2}\right)\right] \cdot 2 \gamma_{21} \psi_{, x z} n_{x} n_{z} \\
& +\psi_{, z} \mathscr{N}_{x}+\mathscr{N}_{z} \psi_{, x}+\delta_{2}\left(\chi_{, x} \mathscr{L}_{, x}+\chi_{, z} \mathscr{L}_{, z}\right) \\
& \delta_{3} \psi_{, x z \tau}=a_{1} \psi_{, z z z z}+a_{2} \psi_{, x z z z}+a_{3} \psi_{, x x z z}+a_{4} \psi_{, x x x z} \\
& +a_{5} \psi_{, x x x x}+a_{6} \psi_{, z z z}+a_{7} \psi_{, x z z}+a_{8} \psi_{, x x z}+a_{9} \psi_{, x x x} \\
& +a_{10} \psi_{, z z}+a_{11} \psi_{, x z}+a_{12} \psi_{, x x}+\mathscr{F}_{,} \\
& \chi_{, \tau}=\left[\chi_{, x}\left(\lambda n_{x}^{2}+n_{z}^{2}\right)+(\lambda-1) n_{x} n_{z} \chi_{, z}\right]_{, x} \\
& +\left[\chi_{, z}\left(\lambda n_{z}^{2}+n_{x}^{2}\right)+(\lambda-1) n_{x} n_{z} \chi_{, x}\right]_{, z} \\
& +\delta_{4} \chi\left(\nabla \cdot \frac{\partial \mathscr{R}^{t m}}{\partial \nabla \chi}\right)-\psi_{, z} \chi_{, x}+\psi_{x} \chi,{ }_{, z}
\end{aligned}
$$

где $\mathscr{M}_{0}=\nabla \cdot \hat{\mathbf{n}}, K_{31}=K_{3} / K_{1}$ - отношение коэффициентов упругости продольного и поперечного изгибов, 
$\gamma_{21}=\gamma_{2} / \gamma_{1}-$ отношение коэффициентов вращательной вязкости, $\bar{\psi}=\frac{t_{T}}{d^{2}} \psi-$ безразмерная функция тока $\psi$ для поля скорости $\mathbf{v}=u \hat{\mathbf{i}}+w \hat{\mathbf{k}}=-\nabla \times \hat{\mathbf{j}} \psi, \tau=\frac{t}{t_{T}}-$ безразмерное время, а $t_{T}=\frac{\rho C_{p} d^{2}}{\lambda_{\perp}}-$ характерное время используемое для нормировки. Выражения для функции $\mathscr{F}=\left(\sigma_{x x}^{e l}+\sigma_{x x}^{t m}-\sigma_{z z}^{e l}-\sigma_{z z}^{t m}\right)_{, x z}+\left(\sigma_{z x}^{e l}+\sigma_{z x}^{t m}\right)_{, z z}$ $-\left(\sigma_{x z}^{e l}+\sigma_{x z}^{t m}\right)_{, x x}$ и коэффициентов $a_{i}(i=1, \ldots, 12)$, а также выражения для термомеханического $\sigma_{i j}^{t m}$ $(i, j=x, z) \quad$ и $\quad$ упругого $\sigma_{i j}^{e l}(i, j=x, z)$ вкладов в полный тензор напряжения $\sigma_{i j} \quad(i, j=x, z)$ даны в Приложении и в ссылке [16]. Вспомогательные функции имеют вид: $f=n_{x, z}-n_{z, x}, n_{z, \tau}=\frac{\partial n_{z}}{\partial \tau}$, $\mathscr{N}_{z}=n_{z} n_{x, z}-n_{x} n_{z, z}, \quad \mathscr{L}_{x}=n_{x} n_{z, x}-\frac{3}{2} n_{z} n_{x, x}+\frac{1}{2} n_{x} n_{x, z}$, $\mathscr{L}_{z}=-n_{z} n_{x, z}+\frac{3}{2} n_{x} n_{z, z}-\frac{1}{2} n_{z} n_{z, x}$. В системе уравнений (8) $-(10)$ и в последующем изложении черта над безразмерной функцией тока опущена. Безразмерный термомеханический вклад в функцию Рэлея $\mathscr{R}^{t m}$ может быть записан в виде (детали изложены в Приложении и в ссылке [16])

$$
\begin{aligned}
& \mathscr{R}^{\mathrm{tm}}=\chi_{, x}\left(-\frac{1}{2} n_{z} \mathscr{M}_{0}-n_{z} M_{x x}\right. \\
& \left.+n_{x}^{2}\left(n_{x} M_{z z}-M_{x x} n_{z}+2 n_{x} M_{x z}\right)\right) \\
& +\chi, z\left(\frac{1}{2} n_{x} \mathscr{M}_{0}+n_{x} M_{z z}+n_{z}^{2}\left(n_{x} M_{z z}-M_{x x} n_{x}-2 n_{z} M_{x z}\right)\right),
\end{aligned}
$$

где $M_{i j}(i, j=x, z)$ - компоненты тензора $\mathbf{M}=$ $=\frac{1}{2}\left[\nabla \hat{\mathbf{n}}+(\nabla \hat{\mathbf{n}})^{T}\right]$. Система уравнений $(8)-(10)$ характеризуется набором параметров $\delta_{1}=\frac{t_{T} K_{1}}{\gamma_{1} d^{2}}, \delta_{2}=\frac{\rho C_{p} T_{N I}}{\lambda_{\perp}} \frac{\xi}{\gamma_{1}}$, $\delta_{3}=\frac{\rho d^{2}}{\gamma_{1} t_{T}}$, и $\delta_{4}=\frac{\xi}{\lambda_{\perp} t_{T}}$. Здесь $\xi=10^{-12}[\mathrm{~J} / \mathrm{mK}]-$ термомеханическая постоянная [12].

Эволюция поля директора и скорости под действием градиента температуры $\nabla \chi$ может быть описана системой нелинейных дифференциальных уравнений в частных производных (8)-(10) и должна быть дополнена граничными условиями, как для поля директора и температуры, так и для функции тока

$$
\begin{gathered}
\left(n_{x}\right)_{x= \pm 10,-1 \leq z \leq 1}=0, \quad\left(n_{x}\right)_{-10<x<10, z=1}=1, \\
\left(n_{x}\right)_{-10<x<-L, z=-1}=\left(n_{x}\right)_{L<x<10, z=-1}=0, \\
\left(n_{x}\right)_{-L<x<L, z=-1}=\sin \mathcal{A}, \\
\chi_{x= \pm 10,-1 \leq z \leq 1}=0.97, \quad \chi_{-10<x<10, z=1}=0.97, \\
\left(\chi_{, z}(x, z, \tau)\right)_{z=-1}=\left[\frac{q_{z}-(\lambda-1) n_{x} n_{z} \chi, x}{\lambda n_{z}^{2}+n_{x}^{2}}\right]_{z=-1}, \\
\left(\psi_{, x}\right)_{x= \pm 10,-1 \leq z \leq 1}=\left(\psi_{, z}\right)_{x= \pm 10,-1 \leq z \leq 1}=0, \\
\left(\psi_{, x}\right)_{-10 \leq x \leq 10, z=-1}=\left(\psi_{, z}\right)_{-10 \leq x \leq 10, z=-1}=0 .
\end{gathered}
$$

В свою очередь, начальное условие для поля директора имеет вид

$$
\hat{\mathbf{n}}(x, z, \tau=0)=\hat{\mathbf{n}}_{\text {elas }}^{e q}(x, z),
$$

в то время как начальные условия для функции тока и температуры могут быть записаны в виде

$$
\psi(x, z, \tau=0)=0,
$$

и

$$
\chi(x, z, \tau=0)=0.97
$$

соответственно.

В случае ЖК-системы, образованной молекулами 4- $n^{\prime}$-пентил- $n^{\prime}$-цианобифенила (5ЦБ), температурный интервал существования нематической фазы соответствует $297 \leq T \leq 308 \mathrm{~K}$. В этом интервале температур величины параметров оцениваются как $\delta_{1} \sim 10^{-3}, \delta_{2} \sim 0.3$, $\delta_{3} \sim 10^{-6}$, и $\delta_{4} \sim 10^{-4}$ соответственно. Поэтому с учетом того, что $\delta_{3} \ll 1$, уравнение (9) принимает вид

$$
\begin{aligned}
a_{1} \psi_{, z z z z} & +a_{2} \psi_{, x z z z}+a_{3} \psi_{, x x z z}+a_{4} \psi_{, x x x z}+a_{5} \psi_{, x x x x} \\
& +a_{6} \psi_{, z z z}+a_{7} \psi_{, x z z}+a_{8} \psi_{, x x z}+a_{9} \psi_{, x x x} \\
& +a_{10} \psi_{, z z}+a_{11} \psi_{, x z}+a_{12} \psi_{, x x}+\mathscr{F}=0 .
\end{aligned}
$$

\section{3. Решение гидродинамических уравнений и основные результаты}

Процесс термомеханического формирования вихревого течения в ГОЖК-канале под действием лазерного излучения сфокусированного на нижней границе канала, содержащей ОД, описывается системой нелинейных дифференциальных уравнений в частных производных (8), (16) и (10) совместно с граничными (12) и начальными условиями, как для поля директора (13) и функции тока (14), так и для температуры (15) соответственно. Здесь равновесное распределение поля директора $\hat{\mathbf{n}}_{\text {elast }}^{\text {eq }}(x, z)$ получено в результате решения уравнения (8) при условии, что отсутствуют течение и градиенты температуры $\psi_{, x}=\psi_{, z}=\chi_{, x}=\chi_{, z}=0$. Граничное условие для поля директора $\hat{\mathbf{n}}_{\text {elast }}^{\mathrm{eq}}(x, z)$ имеет вид

$$
\begin{gathered}
\left(n_{x}\right)_{x= \pm 10,-1 \leq z \leq 1}=0, \quad\left(n_{x}\right)_{-10<x<10, z=1}=1, \\
\left(n_{x}\right)_{-10<x<-L, z=-1}=\left(n_{x}\right)_{L<x<10, z=-1}=0, \\
\left(n_{x}\right)_{-L<x<L, z=-1}=\sin \mathcal{A},
\end{gathered}
$$

а начальное условие может быть записано как

$$
\begin{aligned}
& \theta_{\text {elast }}(x, z, \tau=0) \\
& = \begin{cases}0, & -1 \leq z \leq 1, \quad x= \pm 10, \\
\frac{\pi}{4}(z+1), & -10<x<-L, \quad L<x<10, \\
\frac{\pi}{2}, & z=-1, \quad-L<x<-L .\end{cases}
\end{aligned}
$$

Формирование вихревого течения в ГОЖК-канале под действием лазерного излучения сфокусированного на 


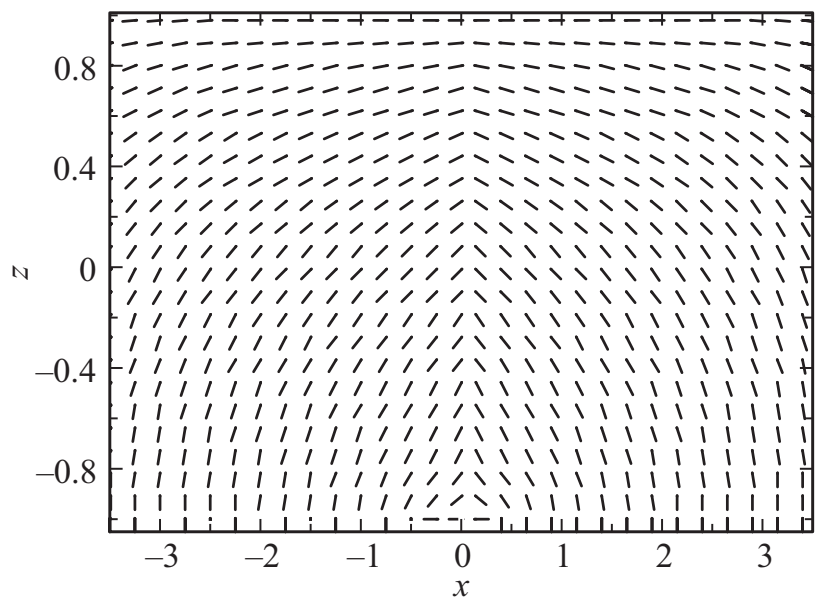

Рис. 1. Фрагмент распределения поля директора $\hat{\mathbf{n}}_{\text {elast }}^{\text {eq }}(x, z)$ в ГОЖК-канале вблизи ОД, локализованного на нижней границе $-L<x<L, z=-1$ канала в начальный момент времени $\tau=0$. Здесь $L= \pm 0.5$.

нижней границе было исследовано численно методом релаксаций [17], а критерием сходимости итерационной процедуры был выбран $\epsilon=\left|\left(\theta_{m+1}-\theta_{m}\right) / \theta_{m}\right| \sim 10^{-4}$. Здесь $m$ - номер итерации.

На рис. 1 представлены результаты расчета распределения поля директора $\hat{\mathbf{n}}_{\text {elast }}^{\text {eq }}(x, z)$ в ГОЖК-канале вблизи ОД, локализованного на нижней границе $(-L<x<L$, $z=-1)$ канала в начальный момент времени $\tau=0$. Здесь $L= \pm 0.5-$ соответствует правой и левой границам ориентационного дефекта соответственно. Согласно расчетам, найбольшее изменение величины $\nabla \theta_{\mathrm{eq}}(x, z)$ достигается вблизи ОД, и его влияние простирается практически на 1/3 объема ЖК-канала, достигая точки с координатой $x=0, z=0.8$. Располагая начальным распределением поля директора $\hat{\mathbf{n}}_{\text {elast }}^{\mathrm{eq}}(x, z)$, с помощью уравнения (10) и условия отсутствия скорости $(\psi)_{x}=(\psi)_{z}=0$, было рассчитано поле температуры $\chi(x, z, \Delta \tau)$ соответствующее следующему шагу по времени $\Delta \tau$. Это, в свою очередь, позволило рассчитать функцию $\mathscr{F}$ и функцию тока $\psi(x, z, \Delta \tau)$. Следующий шаг по времени $\Delta \tau$ для распределения поля директора, скорости и температуры по объему ГОЖК-канала был осуществлен с помощью сеточного метода [18], причем устойчивость численной процедуры определялась из условия

$$
\begin{gathered}
\frac{\Delta \tau}{\delta_{3}}\left(\frac{1}{(\Delta x)^{2}}+\frac{1}{(\Delta z)^{2}}\right) \leq \frac{1}{2}, \\
\frac{3 a_{5}}{(\Delta x)^{4}}-\frac{2 a_{1}}{(\Delta z)^{4}}>0,
\end{gathered}
$$

где $\Delta x$ и $\Delta z$ - приращения пространственных переменных, а коэффициенты $a_{1}$ и $a_{5}$ приведены в Приложении ссылки [16].

Эволюция распределения поля скорости $\mathbf{v}(x, z, \tau)=u(x, z, \tau) \hat{\mathbf{i}}+w(x, z, \tau) \hat{\mathbf{k}}$ в микроразмерном ГОЖК-канале под действием потока тепла q, направленного под углом $\alpha$ к нижней границе канала, содержащего ОД, представлена на рис. 2-4. Для того чтобы понять какую роль играет тепловой поток
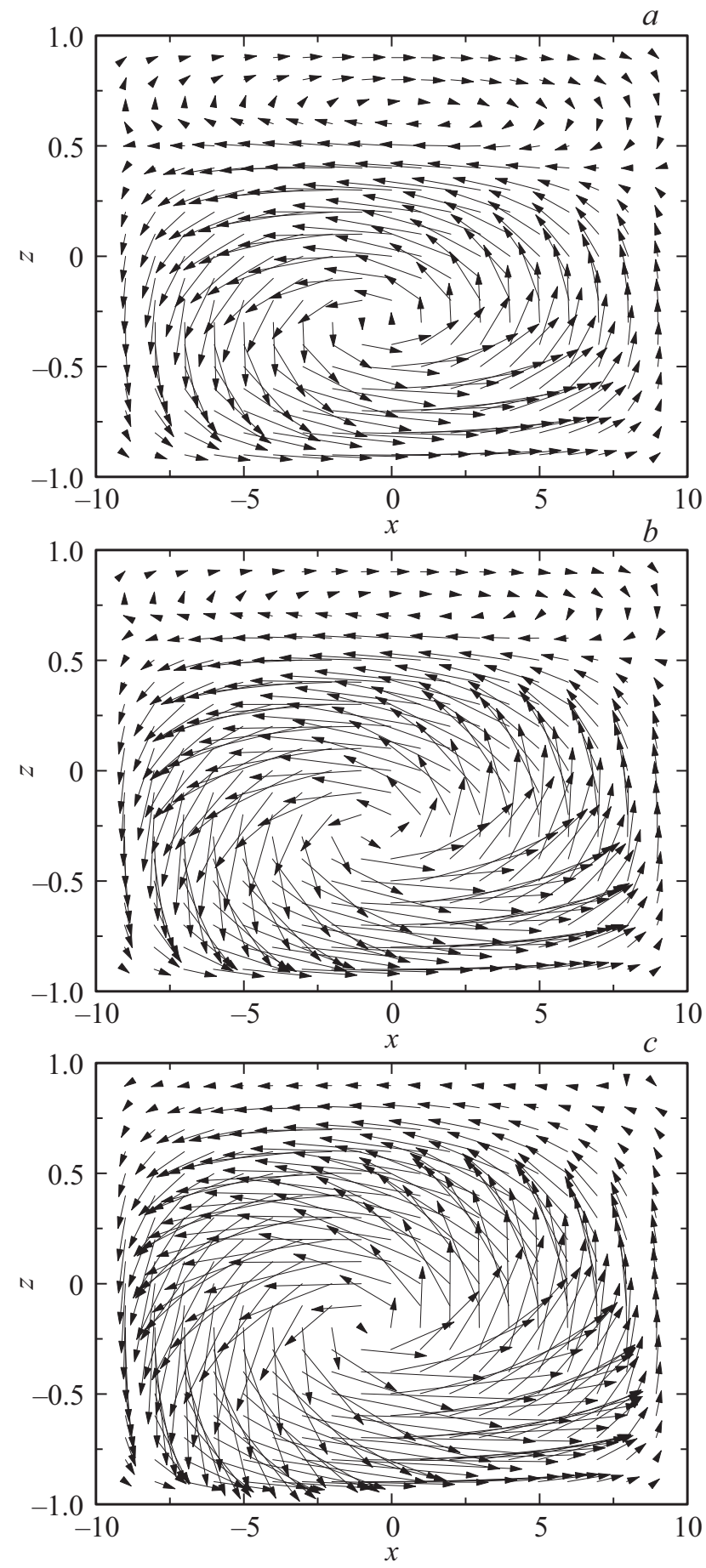

Рис. 2. Эволюция распределения поля скорости $\mathbf{v}(x, z, \tau)=$ $=u(x, z, \tau) \hat{\mathbf{i}}+w(x, z, \tau) \hat{\mathbf{k}}$ в микроразмерном ГОЖК-канале под действием потока тепла $\mathbf{q}$ направленного под углом $\alpha=20^{\circ}$ к нижней границе канала содержащего ОД, для трех значений времени $\tau$ : $(a) \sim 10^{-5}(\sim 18 \mu \mathrm{s}),(b) \sim 4 \cdot 10^{-5}$ $(\sim 72 \mu \mathrm{s})$ и $(c) \tau_{\text {in }} \sim 1.6 \cdot 10^{-4}(\sim 0.29 \mathrm{~ms})$ соответственно. Стрелке длиной в $1 \mathrm{~mm}$ соответствует величина вектора скорости, равная $1.8 \mu \mathrm{m} / \mathrm{s}$. 
$\mathbf{q}=q_{z} \hat{\mathbf{k}}=\mathscr{Q} \sin \alpha \hat{\mathbf{k}}$ в формировании вихревого течения в микроразмерном ГОЖК-канале предположим, что этот поток q формируется под действием направленного лазерного излучения, сфокусированного по всей длине $-L<x<L, z=-1$ ОД на нижней границе ЖК-канала. При этом время накачки $\tau_{\text {in }}$ лазерного излучения мощностью $\mathbb{Q}_{0}=0.05\left(\sim 4.2 \cdot 10^{-4} \mathrm{~mW} / \mu \mathrm{m}^{2}\right)$ равно $1.6 \cdot 10^{-4}(\sim 0.29 \mathrm{~ms})$.
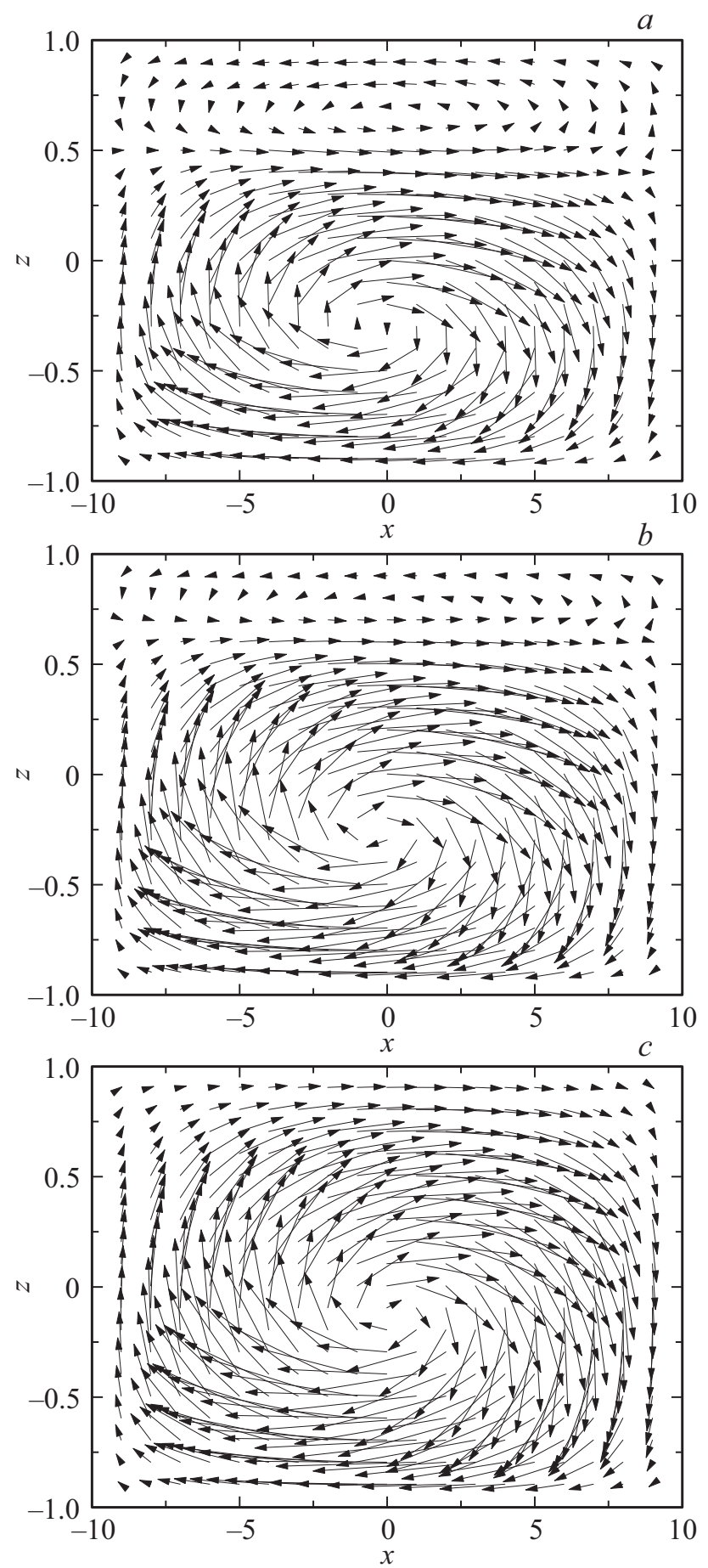

Рис. 3. То же, что на рис. 2, для потока тепла q, направленного под углом $\alpha=160^{\circ}$ к нижней границе канала, содержащего ОД.
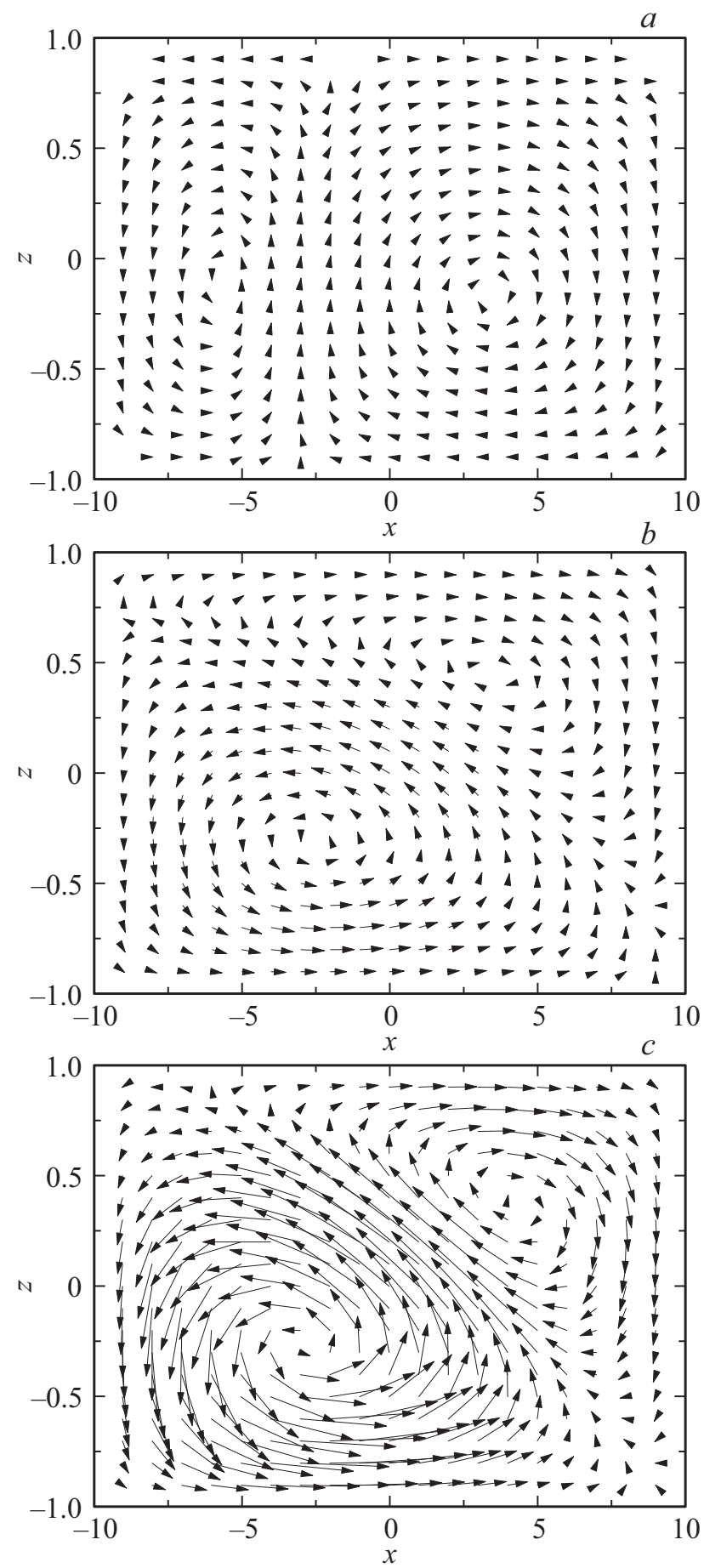

Рис. 4. То же, что на рис. 2 , для потока тепла $\mathbf{q}$, направленного под углом $\alpha=90^{\circ}$ к нижней границе канала, содержащего ОД.

Здесь мощность и продолжительность лазерной накачки подобрана таким образом, чтобы вариация температуры соответствовала области существования нематической фазы. На всех трех рисунках (рис. 2-4) стрелке длиной в $1 \mathrm{~mm}$ соответствует величина вектора скорости, равная $1.8 \mu \mathrm{m} / \mathrm{s}$. Вначале рассмотрим случай когда тепловой поток $\mathbf{q}$ направлен под углом $\alpha=20^{\circ}$ к нижней границе канала содержащего ориентацион- 
ный дефект. На временах порядка $\sim 10^{-5}(\sim 18 \mu s)$ распределение поля скорости $\mathbf{v}(x, z, \tau)$ характеризуется зарождением двух вихрей, вращающихся навстречу друг другу (рис. 2,a). При этом, первый, наиболее сильный вихрь располагается вблизи нижней, более теплой границы ГОЖК-канала, занимая при этом практически три четверти объема ЖК-фазы и вращается против часовой стрелки. Второй, более слабый вихрь, располагается вблизи верхней менее прогретой границы ГОЖК-канала и вращается по часовой стрелке (случай Іа). По мере прогревания нижней границы ГОЖК-канала с ОД, на временах порядка $\sim 4 \cdot 10^{-5}(\sim 72 \mu s)$, нижний, более сильный вихрь немного ускоряется, а верхний, более слабый вихрь, немного замедляется, по сравнению со случаем Іа (рис. 2,b). К моменту выключения лазерного излучения, спустя время $\tau=\tau_{\text {in }} \sim 1.6 \cdot 10^{-4}$ $(\sim 0.29 \mathrm{~ms})$, этот двувихревой гидродинамический поток распадается и окончательно формируется одновихревой поток, направленный против часовой стрелки, с центром вращения в середине ГОЖК-канала (случай I) (рис. 2,c). При этом максимальная величина скорости вращения $v_{\max }(\mathrm{I}) \sim 0.1 \mathrm{~mm} / \mathrm{s}$ достигается вблизи ОД, который разогревается до температуры $\chi_{-L<x<L, z=-1}=0.991$ $(\sim 304.5 \mathrm{~K})$. При смене направления потока тепла $\mathbf{q}=q_{z} \hat{\mathbf{k}}=\mathscr{Q} \sin \alpha \hat{\mathbf{k}}$ через нижнюю границу ГОЖК-канала с ОД, со значения $\alpha=20^{\circ}$ на $160^{\circ}$, распределение поля скорости $\mathbf{v}(x, z, \tau)$ характеризуется также зарождением двух вихрей, направленных навстречу друг другу, но при этом меняется направленность вихрей (рис. 3,a). На временах порядка $\sim 10^{-5}(\sim 18 \mu \mathrm{s})$, первый, найболее сильный вихрь вращается по часовой стрелке, а второй, слабый вихрь - против часовой стрелки. Сильный вихрь, как и в случае I, расположен вблизи более теплой нижней границы ГОЖК-канала, а слабый вихрь, вблизи верхней границы канала (рис. 3,a). Дальнейшая эволюция двувихревого течения в ГОЖК-канале, на временах порядка $\sim 4 \cdot 10^{-5}(\sim 72 \mu \mathrm{s})$, повторяет эволюцию гидродинамического течения характерного для случая I (рис. $3, b$ ), с той особенностью, что спустя время $\tau=\tau_{\text {in }} \sim 1.6 \cdot 10^{-4}(\sim 0.29 \mathrm{~ms})$, окончательно сформировавшийся одновихревой поток вращается по часовой стрелке, с центром вращения в середине ГОЖК-канала (случай II) (рис. 3,c). При этом максимальная величина скорости вращения $v_{\max }(\mathrm{II}) \sim 0.1 \mathrm{~mm} / \mathrm{s}$ достигается вблизи ОД, который также, как и в случае I, разогревается до температуры $\chi_{-L<x<L, z=-1}=0.991(\sim 304.5 \mathrm{~K})$. Эволюция распределения поля скорости $\mathbf{v}(x, z, \tau)$ в микроразмерном ГОЖК-канале под действием потока тепла q направленного перпендикулярно $\left(\alpha=90^{\circ}\right)$ нижней границе ГОЖК-канала с ОД, отличается от двух вышеописанных сценариев эволюции поля скорости. На временах порядка $\sim 10^{-5}(\sim 18 \mu \mathrm{s})$, распределение поля скорости $\mathbf{v}(x, z, \tau)$ также, как и в случаях I и II, характеризуется зарождением двух вихрей, направленных навстречу друг другу (рис. 4,a). При этом, первый, найболее крупный вихрь располагается в правой части ГОЖК-канала и вращается по часовой стрелке, занимая при этом практически три четверти объема ЖК-фазы.
Второй, более мелкий вихрь, располагается в левой части ГОЖК-канала и вращается против часовой стрелки (рис. 4,a). При этом, скорости вращения этих вихрей практически равны друг другу. По мере прогревания нижней границы ГОЖК-канала с ОД, на временах порядка $\sim 4 \cdot 10^{-5}(\sim 72 \mu \mathrm{s})$, размер мелкого левого вихря начинает расти и занимает большую часть ЖК-объема вблизи нижней более теплой границы ГОЖК-канала (рис. $4, b)$. Скорость вращения левого, теперь уже более крупного вихря возрастает, в то время как скорость вращения правого вихря практически не меняется. К моменту выключения лазерного излучения, спустя время $\tau=\tau_{\text {in }} \sim 1.6 \cdot 10^{-4}(\sim 0.29 \mu \mathrm{s})$, окончательно формируется гидродинамический поток, характеризующийся двумя вихрями, направленными навстречу друг другу (случай III) (рис. 4,c). При этом максимальная величина скорости вращения $v_{\max }$ (III) левого, более крупного вихря, направленного по часовой стрелке, примерно в два раза меньше максимальной скорости вращения обоих вихрей $v_{\max }(\mathrm{I})$ и $v_{\max }(\mathrm{II})$, соответствующих случаям $\mathrm{I}$ и II соответственно.

Основываясь на наших вычислениях, можно сделать вывод, что направление теплового потока q через нижнюю границу ГОЖК-канала содержащего ОД может кардинальным образом влиять на формирование поля скорости в микроразмерном ГОЖК-объеме с ориентационным дефектом. Под влиянием теплового потока, инициируемого сфокусированным лазерным излучением, в ГОЖК-канале с ОД может формироваться как одно, так и двувихревой поток.

\section{4. Заключение}

В предлагаемой работе численными методами, в рамках нелинейного обобщения классической теории Эриксена-Лесли, с учетом баланса энтропии, описаны несколько сценариев формирования вихревых течений в микроразмерном гибридно-ориентированном жидкокристаллическом (ГОЖК) канале с ориентационным дефектом (ОД) под действием сфокусированного лазерного излучения. Учет термомеханических вкладов в выражении для сдвигового напряжения и в уравнении баланса энтропии позволил описать процесс формирования двувихревого потока в ГОЖК-каналах. Было установлено, что под действием потока тепла q направленного под углом $\alpha$ к нижней границе ГОЖК-канала, содержащего ОД, могут окончательно сформироваться два либо один вихрь, хотя на начальном этапе формирования вихревых потоков всегда зарождаются два вихря, вращающиеся навстречу друг другу.

Исследованные в работе особенности, связанные с реакцией ЖК-материала, инкапсулированного в тонкие и сверхтонкие каналы на локально формирующиеся градиенты температуры, необходимо учитывать при создании сенсоров и датчиков, используемых в биотехнологических приложениях, медицине и биометрических оптических системах. 


\section{5. Приложение}

Уравнение баланса моментов действующих на единицу объема ЖК-фазы имеет вид

$$
\mathbf{T}_{\mathrm{el}}+\mathbf{T}_{\mathrm{vis}}+\mathbf{T}_{\mathrm{tm}}=0
$$

где $\mathbf{T}_{\mathrm{el}}=\frac{\delta \mathscr{W}_{\mathrm{el}}}{\delta \hat{\mathbf{n}}} \times \hat{\mathbf{n}}-$ упругий, $\mathbf{T}_{\mathrm{vis}}=\frac{\delta \mathscr{R}^{\mathrm{vis}}}{\delta \hat{\mathbf{n}}_{t}} \times \hat{\mathbf{n}}-$ вязкий и $\mathbf{T}_{\mathrm{tm}}=\frac{\delta \mathscr{R}^{\mathrm{tm}}}{\delta \hat{\mathbf{n}}_{t}} \times \hat{\mathbf{n}}-$ термомеханический вклады в баланс моментов соответственно. Здесь $\hat{\mathbf{n}}_{t} \equiv \frac{d \hat{\mathbf{n}}}{d t}-$ материальная производная вектора $\hat{\mathbf{n}}=n_{x} \hat{\mathbf{i}}+n_{z} \hat{\mathbf{k}}$.

Уравнение баланса линейных моментов, действующих на единицу объема ЖК-фазы имеет вид

$$
\rho \frac{d \mathbf{v}}{d t}=\nabla \cdot \sigma
$$

где $\frac{d v}{d t}=\frac{\partial v}{\partial t}+u v_{, x}+w v_{, z}, \quad \sigma=\sigma^{\mathrm{el}}+\sigma^{\mathrm{vis}}+\sigma^{\mathrm{tm}}-\mathscr{P} \mathscr{E}-$ полное выражение для тензора напряжения (ТН), состоящее из упругого $\sigma^{\mathrm{el}}=-\frac{\partial \mathscr{W}_{\mathrm{el}}}{\partial \nabla \hat{\mathbf{n}}} \cdot(\nabla \hat{\mathbf{n}})^{T}$, вязкого $\sigma^{\text {vis }}=\frac{\delta \mathscr{R}^{\text {vis }}}{\delta \nabla \mathbf{v}}$ и термомеханического $\sigma^{\mathrm{tm}}=\frac{\delta \mathscr{R}^{\mathrm{tm}}}{\delta \nabla \mathbf{v}}$ вкладов в ТН соответственно. Здесь $\mathscr{R}=\mathscr{R}^{\text {vis }}+\mathscr{R}^{\text {tm }}+\mathscr{R}^{\text {th }}-$ полная диссипационная функция Рэлея, $\mathscr{W}_{\mathrm{el}}=\frac{1}{2}\left[K_{1}(\nabla \cdot \hat{\mathbf{n}})^{2}\right.$ $\left.+K_{3}(\hat{\mathbf{n}} \times \nabla \times \hat{\mathbf{n}})^{2}\right]-$ плотность упругой энергии, $K_{1}$ и $K_{3}$ - продольный и поперечный коэффициенты упругости, $\mathscr{P}$ - гидростатическое давление в ЖК-системе, $\mathscr{E}$ - единичный тензор, в то время как

$$
\begin{aligned}
\mathscr{R}^{\mathrm{vis}}= & \alpha_{1}\left(\hat{\mathbf{n}} \cdot \mathbf{D}_{s} \cdot \hat{\mathbf{n}}\right)^{2}+\gamma_{1}\left(\hat{\mathbf{n}}_{t}-\mathbf{D}_{a} \cdot \hat{\mathbf{n}}\right)^{2} \\
& +2 \gamma_{2}\left(\hat{\mathbf{n}}_{t}-\mathbf{D}_{a} \cdot \hat{\mathbf{n}}\right) \cdot\left(\mathbf{D}_{s} \cdot \hat{\mathbf{n}}-\left(\hat{\mathbf{n}} \cdot \mathbf{D}_{s} \cdot \hat{\mathbf{n}}\right) \hat{\mathbf{n}}\right) \\
& +\alpha_{4} \mathbf{D}_{s} \mathbf{D}_{s}+\left(\alpha_{5}+\alpha_{6}\right)\left(\hat{\mathbf{n}} \cdot \mathbf{D}_{s} \cdot \mathbf{D}_{s} \cdot \hat{\mathbf{n}}\right)
\end{aligned}
$$

— вязкий, а

$$
\begin{aligned}
\frac{1}{\xi} \mathscr{R}^{\mathrm{tm}} & =(\hat{\mathbf{n}} \cdot \nabla T) \mathbf{D}_{s}: \mathbf{M}+\nabla T \cdot \mathbf{D}_{s} \cdot \mathbf{M} \cdot \hat{\mathbf{n}}+(\hat{\mathbf{n}} \cdot \nabla T) \\
& \times\left(\hat{\mathbf{n}}_{t}-\mathbf{D}_{a} \cdot \hat{\mathbf{n}}-3 \mathbf{D}_{s} \cdot \hat{\mathbf{n}}+3\left(\hat{\mathbf{n}} \cdot \mathbf{D}_{s} \cdot \hat{\mathbf{n}}\right) \hat{\mathbf{n}}\right) \cdot \mathbf{M} \cdot \hat{\mathbf{n}} \\
& +\hat{\mathbf{n}}(\nabla \mathbf{v})^{T} \cdot \mathbf{M} \cdot \nabla T+\frac{1}{2}\left(\hat{\mathbf{n}} \cdot \mathbf{D}_{s} \cdot \hat{\mathbf{n}}\right) \nabla T \cdot \mathbf{M} \cdot \hat{\mathbf{n}} \\
& +\hat{\mathbf{n}}_{t} \cdot \mathbf{M} \cdot \nabla T+\frac{1}{2} \mathscr{M}_{0} \nabla T \cdot \nabla \mathbf{v} \cdot \hat{\mathbf{n}} \\
& +(\hat{\mathbf{n}} \cdot \nabla T) \mathscr{M}_{0}\left(\hat{\mathbf{n}} \cdot \mathbf{D}_{s} \cdot \hat{\mathbf{n}}\right)+\frac{1}{2} \mathscr{M}_{0} \hat{\mathbf{n}}_{t} \cdot \nabla T
\end{aligned}
$$

и

$$
\mathscr{R}^{\text {th }}=\frac{1}{T}\left(\lambda_{\|}(\hat{\mathbf{n}} \cdot \nabla T)\right)^{2}+\lambda_{\perp}\left(\nabla T-\hat{\mathbf{n}}(\hat{\mathbf{n}} \cdot \nabla T)^{2}\right)
$$

- термомеханический и термический вклады в полное выражение для функции Рэлея $\mathscr{R}$ соответственно. Здесь $\alpha_{1} \div \alpha_{6}-$ коэффициенты вязкости Лесли, $\gamma_{1}(T)$ и $\gamma_{2}(T)-$ коэффициенты вращательной вязкости ЖКсистемы, $\xi$ - термомеханическая постоянная, $\lambda_{\|}$и $\lambda_{\perp}-$ коэффициенты теплопроводности ЖК-системы вдоль и поперек направления директора $\hat{\mathbf{n}}$ соответственно. Тензоры $\quad \mathbf{D}_{s}=\frac{1}{2}\left[\nabla \mathbf{v}+(\nabla \mathbf{v})^{T}\right]$ и $\quad \mathbf{D}_{a}=\frac{1}{2}\left[\nabla \mathbf{v}-(\nabla \mathbf{v})^{T}\right]-$ являются симметричным и асимметричным вкладами в тензор скорости деформации, $\mathbf{M}=\frac{1}{2}\left[\nabla \hat{\mathbf{n}}+(\nabla \hat{\mathbf{n}})^{\mathrm{T}}\right]$ и $\mathscr{M}_{0}=\nabla \cdot \hat{\mathbf{n}}-$ скалярный инвариант тензора $\mathbf{M}$.

Уравнение теплопроводности описывающее изменение поля температуры $T(x, z, t)$ под действием потока тепла q через нижнюю границу ГОЖК-канала имеет вид

$$
\rho C_{P} \frac{d T}{d t}=-\nabla \cdot \mathbf{Q},
$$

где $\mathbf{Q}=-T \frac{\delta \mathscr{R}}{\delta \nabla T}-$ есть тепловой поток в ЖК-системе, а $C_{P}$ - коэффициент теплоемкости соответственно.

\section{Список литературы}

[1] H.A. Stone, A.D. Stroock, A. Ajdari. Annu. Rev. Fluid Mech. 36, 381 (2004).

[2] T.M. Squires, S.R. Quake. Rev. Mod. Phys. 77, 977 (2005).

[3] R.B. Shoch, J.Y. Han, P. Renaud. Rev. Mod. Phys. 80, 839 (2008).

[4] P. Hanggi, F. Marchesoni. Rev. Mod. Phys. 81, 387 (2009).

[5] W. Sparreboom, A. van den Berg, J.C.T. Eijkel. New. J. Phys. 12, 0115004 (2010).

[6] P. Popov, L.W. Hanaker, M. Mirheydari, E.K. Mann, A. Jakli. Sci. Rep. 7, 1603 (2017).

[7] J. Beekman, I. Nys, O. Willekens, K. Neyts. J. Appl. Phys. 121, 023106 (2017).

[8] S. Lee, R. An, J.A. Hunt. Nature Nanotech. 5, 412 (2010).

[9] E. Verneuil, M.L. Cordero, F. Gallaire, Ch.N. Baroud. Langmuir 25, 5127 (2009).

[10] P.G. de Gennes, J. Prost. The Physics of Liquid Crystals. Oxford Univ. Press, Oxford (1995). 400 p.

[11] A.V. Zakharov, A.A. Vakulenko. J. Chem. Phys. 127, 084907 (2007).

[12] R.S. Akopyan, R.B. Alaverdian, E.A. Santrosian, Y.S. Chilingarian. J. Appl. Phys. 90, 3371 (2001).

[13] J.L. Ericksen. Arch. Ration. Mech. Anal. 4, 231 (1960).

[14] F.M. Leslie. Arch. Ration. Mech. Anal. 28, 265 (1968).

[15] С. Гроот, П. Мазур. Неравновесная термодинамика. Мир, M. (1964). $456 \mathrm{c}$.

[16] A.V. Zakharov, P.V. Maslennikov. Phys. Rev. E 96, 052705 (2017).

[17] И.С. Березин, Н.Р. Жидков. Методы вычислений. Физматгиз, М. (1964). 464 с.

[18] А.А. Самарский, Е.С. Николаев. Методы решения сеточных уравнений. Наука, М. (1978). 592 с.

Редактор Ю.Э. Китаев 